\title{
D Sınıfı Eviricinin Runge-Kutta Metodu ile Analizinin Yapılması
}

\author{
${ }^{1}$ Selim ÖNCÜ, $* 2$ Kenan ÜNAL ve ${ }^{3}$ Uğur TUNÇER \\ ${ }^{1}$ Karabük Üniversitesi, Mühendislik Fakültesi, Elektrik Elektronik Mühendisliği Bölümü \\ *22Gazi Üniversitesi, TUSAŞ Kazan Meslek Yüksekokulu, Motorlu Araçlar ve Ulaştırma Teknolojileri Bölümü \\ ${ }^{3}$ Hitit Üniversitesi, Osmancık Ömer Derindere Meslek Yüksekokulu, Elektronik ve Otomasyon Bölümü
}

\section{Özet}

D sınıfı eviriciler geniş kontrol aralığına sahip olmaları, az sayıda güç anahtarı ve anahtar sürücü devre elemanları gerektirmeleri ve yüksek güçleri kontrol edebilmeleri sebebiyle günümüzde birçok uygulamada kullanılmaktadır. Bu çalışmada D sınıfı rezonans evirici analizi Runge-Kutta algoritması ile gerçekleştirilmiştir. Yapılan analizin sonucunda evirici akımı ve gerilimi, kondansatör gerilimi gibi devre parametrelerine ait grafikler çizdirilmiştir. Aynı devre parametreleri MATLAB Simulink üzerinde yapılan benzetim çalışmasında da tekrarlanarak sonuçlar karşılaştırılmış ve Runge-Kutta algoritmasının doğruluğu ortaya konulmuştur.

Anahtar Kelimeler: Runge-Kutta, D sınıfı evirici, MATLAB Simulink

\begin{abstract}
Class D inverters are used in many applications today because they have a wide control range, require less power switches and switch driver circuit elements, and can control high powers. In this study, class D resonance inverter analysis was carried out with the Runge-Kutta algorithm. As a result of the analysis, graphs of circuit parameters such as inverter current and voltage, capacitor voltage were drawn. The same circuit parameters were repeated in the simulation study on MATLAB Simulink and the results were compared and the accuracy of the Runge-Kutta algorithm was demonstrated.
\end{abstract}

Key Words: Runge-Kutta, Class D inverter, MATLAB Simulink

\section{Giriş}

Güç dönüştürücü devrelerin anahtarlanması için sert anahtarlama [1] veya yumuşak anahtarlama [2-4] teknikleri kullanılabilir. Bir güç dönüştürücü devreden beklenilen yüksek verim, düşük maliyet ve küçük hacimdir [4]. Sert anahtarlama teknikleri kullanılan bir güç dönüştürücüde yüksek anahtarlama frekansı anahtarlama kayıplarının artması nedeniyle verimin düşmesine, yüksek Elektromanyetik Girişime (EMI) ve anahtarlar üzerinde stres oluşmasına neden olur $[1,5,6]$. Sert anahtarlama teknikerinden kaynaklanan bu olumsuzlukları azaltarak anahtarlama frekansının artırılmasının bir yolu ise rezonans güç dönüştürücüleri kullanmaktır [5,7]. Rezonans güç dönüştürücüleri ile anahtarların iletim ve yalıtım durumlarına geçmeleri sırasında üzerindeki gerilim veya akım sıfırlanarak yumuşak anahtarlama gerçekleştirilir [8]. Rezonans güç dönüştürücülerinin kullanılması sayesinde anahtarlama kayıpları olmadan ve EMI azaltılarak güç dönüştürücü devre çalıştırılabilir [9]. Aynı zamanda rezonans devre ile güç dönüştürücü çıkışındaki

*Corresponding author: Address: Gazi University TUSAŞ-Kazan Vocational School. E-mail address: kenanunal@gazi.edu.tr, Phone: +903128000614 
istenmeyen harmonik bileşenler süzülerek kare dalga akım sinyali yerine sinüzoidal bir akım sinyali elde edilir [4]. Bu tip devrelere seri rezonans güç dönüştürücüleri denilmektedir. D sınıfı seri rezonans evirici devre yapısı ise birbirine seri bağlı iki güç anahtarının ortak bağlantı noktalarına seri bağlanmış direnç, endüktans ve kapasitanstan oluşur. Bu evirici devrenin durum denklemleri endüktans ve kapasitanstan kaynaklı ikinci dereceden diferansiyel denklemler içerir [10]. Pratikte diferansiyel denklemlerin analizinde analitik çözüm yöntemleri kullanılır. Bu yüzden rezonans devrenin analizinde doğruluk payının yüksek olması ve hızlı yanıt vermesi nedeniyle Runge-Kutta (RK) yöntemi tercih edilir [10-12].

$\mathrm{Bu}$ çalışmada, D sınıfı seri rezonans eviricinin analizini kolaylaştırmak için Runge-Kutta algoritması kullanılmıştır. Aynı zamanda MATLAB Simulink ortamında benzetim çalışması yapılarak analiz çalışmasının sonuçları doğrulanmıştır.

\section{D Sınıfı Seri Rezonans Evirici}

D sınıfı seri rezonans evirici ve eşdeğer devresi Şekil 1a'da görüldüğ̈ gibi yapısında iki adet ters paralel diyotlu güç anahtarı, direnç, endüktans ve kapasitans bulunmaktadır.

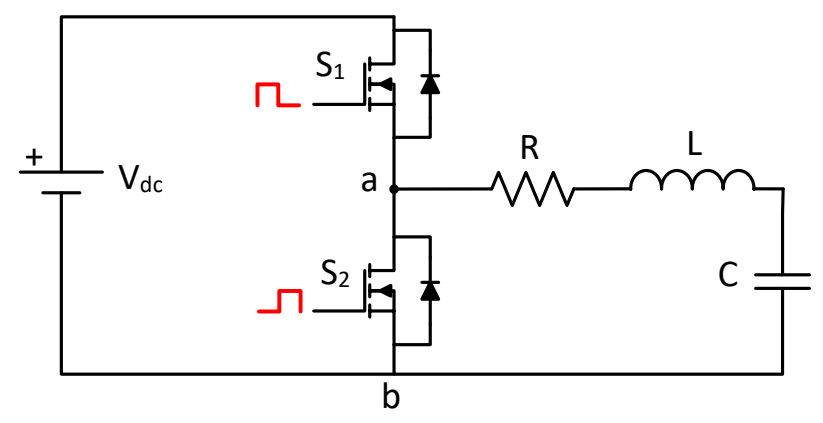

(a)

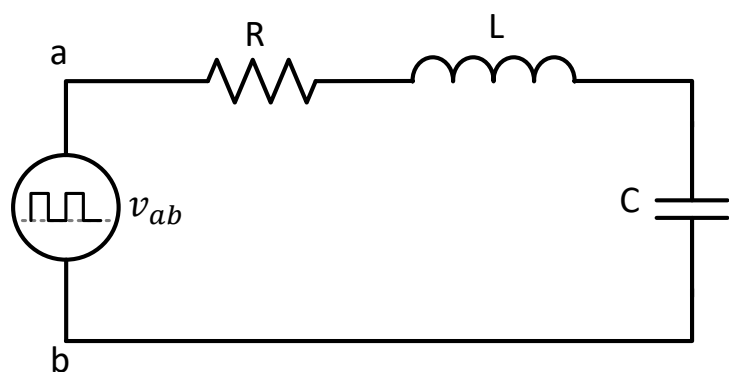

(b)

Şekil 1. (a) D Sınıfı Seri Rezonans Evirici, (b) D Sınıfı Seri Rezonans Evirici Eşdeğer Devresi

D sınıfı eviricilerde bulunan $S_{1}$ ve $S_{2}$ anahtarlarından biri iletimdeyken diğeri kesim durumunda çalışmaktadır [13]. D sınıfı evirici a-b noktaları arasındaki gerilimin denklemi $\left(v_{a b}\right)$ Eşitlik 1'deki gibidir [13]. Eşitlik 1'de $V_{d c}$ kaynak gerilimini, $T$ periyodu ifade etmektedir. Bu durumda eviricinin eşdeğer devresi Şekil 1b'deki gibi olur.

$$
v_{a b}(t)=\left\{\begin{array}{cc}
V_{d c}, & 0<t \leq T / 2 \\
0, & T / 2<t \leq T
\end{array}\right\}
$$


D sınıfı seri rezonans evirici devrenin akımı ( $i$ ) ise Eşitlik 2 ile verilmiştir. Bu eşitlikte $I_{\max }$ evirici akımın tepe değerini, $\theta$ ise faz açısını, $\omega$ açısal frekansı ifade etmektedir. Evirici devre rezonans frekansında çalıştırıldığında faz açısı sıfır olmaktadır.

$$
i(t)=I_{\max } \sin (\omega t-\theta)
$$

Eviricinin rezonans frekansı $\left(f_{r}\right)$ ise Eşitlik 3 ile hesaplanabilir [14].

$$
f_{r}=1 /(2 \pi \sqrt{L C})
$$

\subsection{Runge-Kutta Yöntemi}

Runge-Kutta yöntemleri, yüksek dereceli diferansiyel denklemlerin analizinde Taylor serisi yaklaşımını doğrulayan yaklaşık bir çözüm sunar. Runge-Kutta ailesinin en çok kullanılan yöntemi "Klasik Runge-Kutta yöntemi" olarak adlandırılan dördüncü dereceden Runge-Kutta (RK-4) yöntemidir [10,15]. Bu yöntemin genel formu Eşitlik 4 ile ifade edilir [15].

$$
\left.y_{i+1}=y_{i}+(1 / 6)\left(k_{1}+2 k_{2}+2 k_{3}+k_{4}\right)\right) h
$$

Eşitlik 4'te hesaplanan denklemin yeni değeri $\left(y_{i+1}\right)$, denklemin mevcut değerine $\left(y_{i}\right)$ adım aralığının büyüklüğü (h) ve tahmini eğimin çarpımının eklenmesiyle elde edilir. Burada $i=0,1,2$.. indis numaraların $, k_{1}, k_{2}, k_{3}$ ve $k_{4}$

$$
\begin{aligned}
& k_{1}=f\left(x_{i}, y_{i}\right) \\
& k_{2}=f\left(x_{i}+1 / 2 h, y_{i}+1 / 2 k_{1} h\right) \\
& k_{3}=f\left(x_{i}+1 / 2 h, y_{i}+1 / 2 k_{2} h\right) \\
& k_{4}=f\left(x_{i}+h, y_{i}+k_{3} h\right)
\end{aligned}
$$


RK-4 iterasyonlarını ifade eder. Seri rezonans evirici için durum denklemleri Eşitlik 9 ve 10'da verilmiştir. Durum değişkenleri kapasitör gerilimi $\left(v_{C}\right)$ ve bobin akımı $\left(i_{L}\right)^{\prime}$ dir.

$$
\begin{aligned}
& d v_{c} / d t=1 / C\left(i_{L}\right) \\
& d i_{L} / d t=1 / L\left(V_{d c}-v_{c}-R i_{L}\right)
\end{aligned}
$$

Runge-Kutta metoduna göre rezonans kolunun gerilim ve akım ifadeleri sırasıyla " $k_{1-4}$ serisi" ve “ $j_{1-4}$ serisi” olarak dört iterasyona bağlıdır. Bu iterasyonlar aşağıdaki eşitliklerde verilmiştir.

$$
\begin{aligned}
& k_{1}=(1 / C)\left(i_{L}\right) \\
& j_{1}=(1 / L)\left(V_{d c}-\left(v_{c}\right)-R\left(i_{L}\right)\right) \\
& k_{2}=(1 / C)\left(i_{L}+0.5(h)\left(j_{1}\right)\right) \\
& j_{2}=(1 / L)\left(V_{d c}-\left(v_{c}+0.5(h)\left(k_{1}\right)\right)-R\left(i_{L}+0.5(h)\left(j_{1}\right)\right)\right) \\
& k_{3}=(1 / C)\left(i_{L}+0.5(h)\left(j_{2}\right)\right) \\
& j_{3}=(1 / L)\left(V_{d c}-\left(v_{c}+0.5(h)\left(k_{2}\right)\right)-R\left(i_{L}+0.5(h)\left(j_{2}\right)\right)\right) \\
& k_{4}=(1 / C)\left(i_{L}+(h)\left(j_{3}\right)\right) \\
& j_{4}=(1 / L)\left(V_{d c}-\left(v_{c}+(h)\left(k_{3}\right)\right)-R\left(i_{L}+(h)\left(j_{3}\right)\right)\right)
\end{aligned}
$$

RK-4 yöntemiyle elde edilen seri rezonans devresinin gerilim ve akım denklemleri Eşitlik 19 ve 20 'de sunulmuştur.

$$
\begin{aligned}
& v_{c}(i+1)=v_{c}(i)+h\left(\left(k_{1}+2\left(k_{2}+k_{3}\right)+k_{4}\right) / 6\right) \\
& i_{L}(i+1)=i_{L}(i)+h\left(\left(j_{1}+2\left(j_{2}+j_{3}\right)+j_{4}\right) / 6\right)
\end{aligned}
$$


Bu çalışmada analiz yapılırken kondansatör gerilimi ve evirici akımının ilk enerjileri sıfır alınıp, RK-4 algoritmasında kullanılan adım aralığı anahtarlama periyodunun 1/500'ü olarak seçilmiştir. Runge-Kutta yöntemi ile evirici analizinin gerçekleştirilmesinde Şekil 2'de görülen algoritmadaki adımlar takip edilerek MATLAB M-file kodu yazılmıştır.

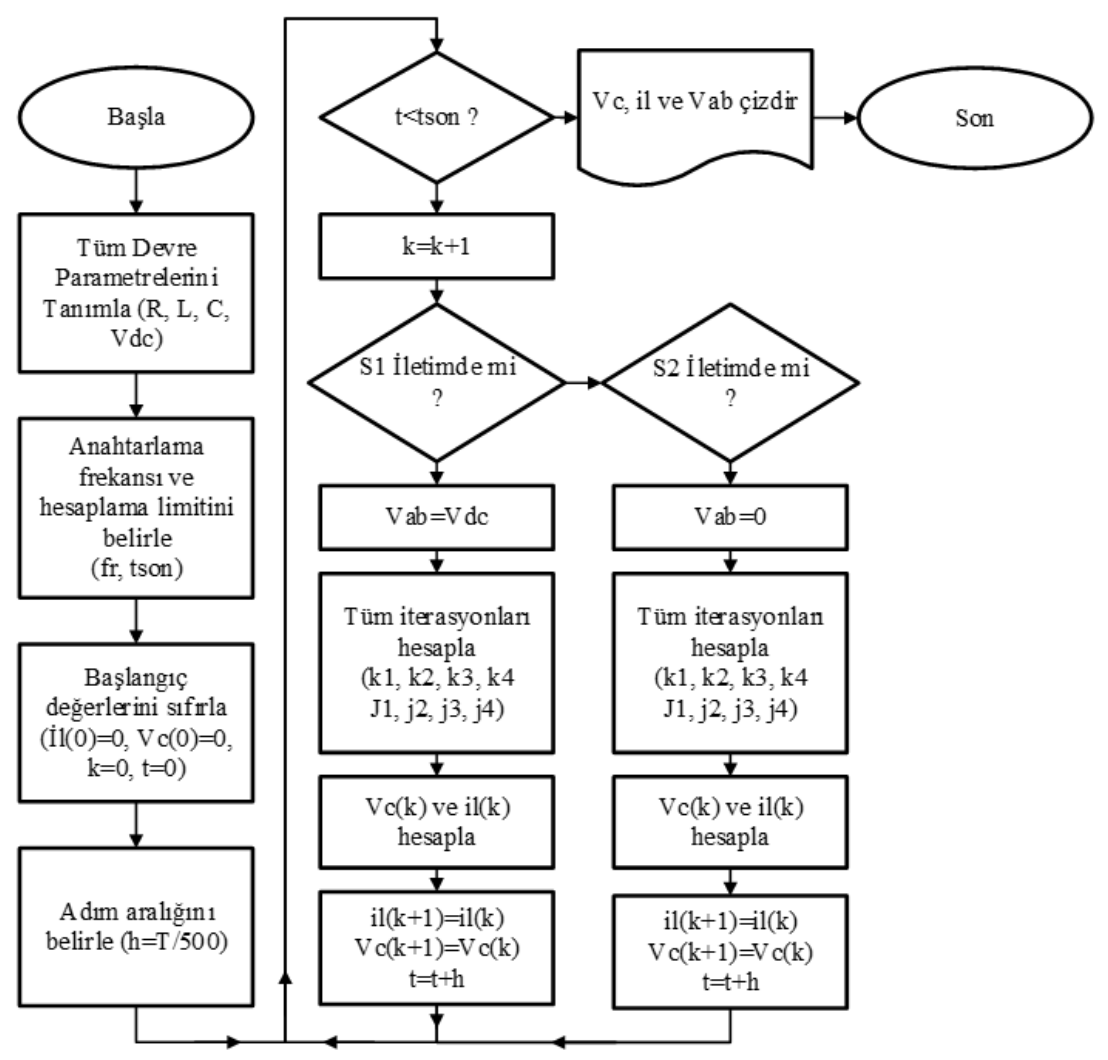

Şekil 2. RK-4 Algoritmas1

\section{Analiz ve Benzetim Çalışması}

D sınıfı seri rezonans evirici devresinin benzetim çalışması Şekil 3'te görüldüğü gibi MATLAB Simulink' te yapılmıştır. Hem benzetim hem de analiz çalışmasında kullanılan devre parametreleri aynı olup, $\mathrm{R}=5,73 \Omega, \mathrm{L}=0,359 \mathrm{mH}, \mathrm{C}=44,8 \mathrm{nF}$ ve $\mathrm{V}_{\mathrm{dc}}=50 \mathrm{~V}$ olarak seçilmiştir. 


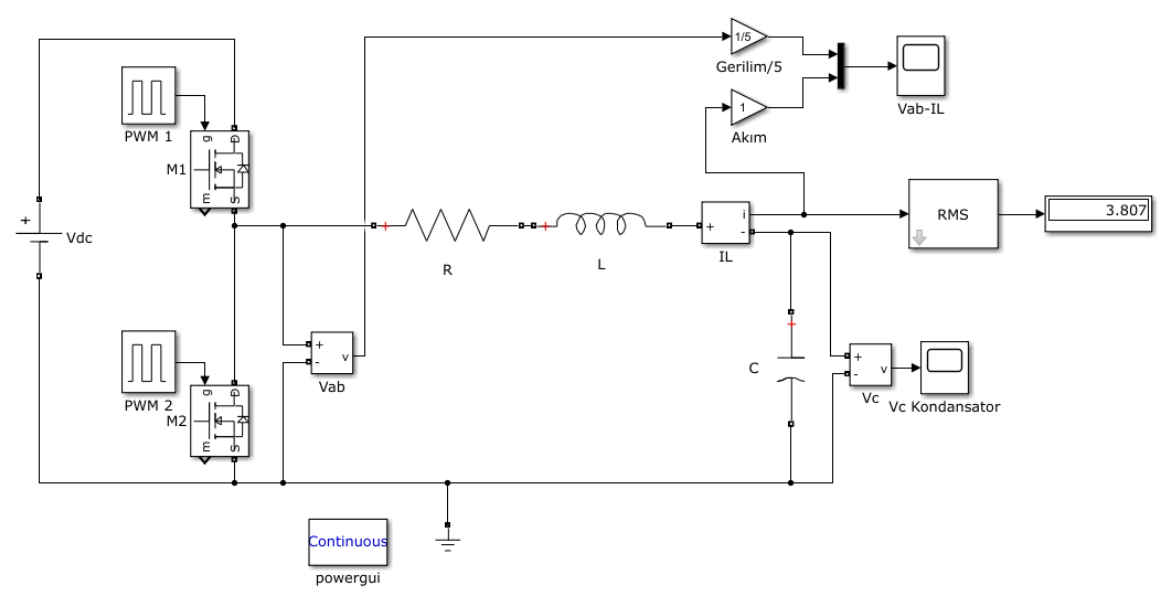

Şekil 3. Benzetim Çalışması

Verilen adımlar takip edilerek yazılan RK-4 kodunun evirici akımı ve gerilimi sonuçları Şekil $4 a$ 'da görülmektedir.

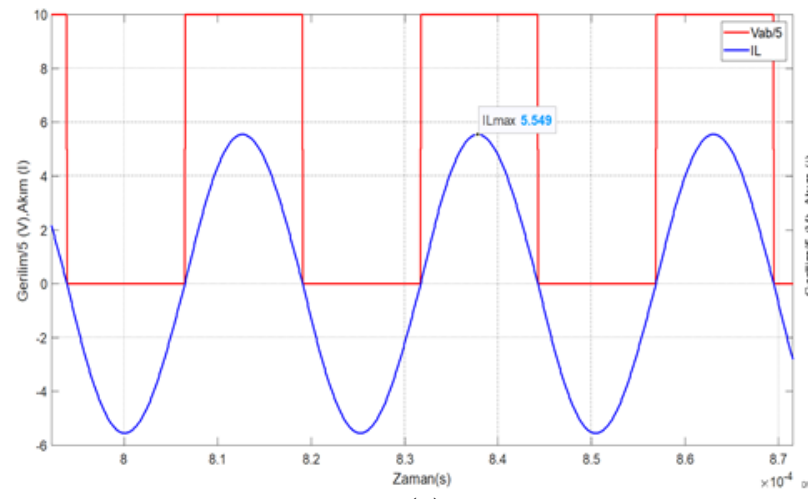

(a)

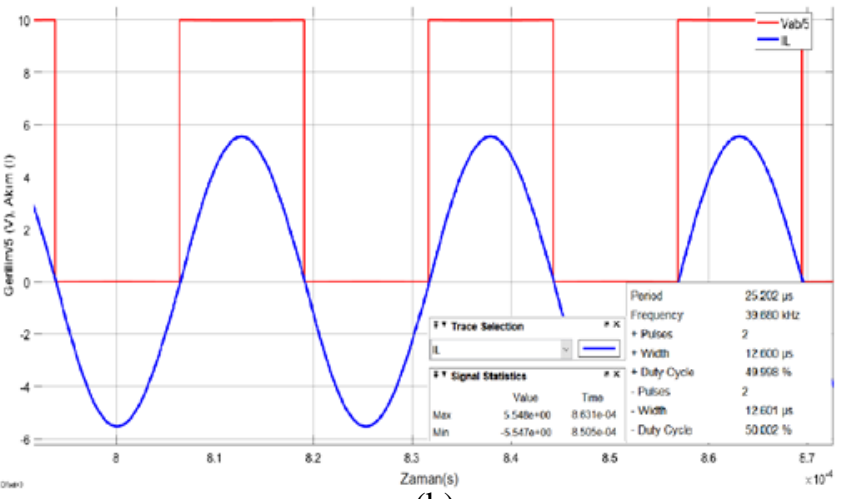

(b)

Şekil 4. Evirici Akımı (a) RK-4 Analizi Sonucu, (b) Benzetim Çalışması Sonucu

RK-4 analizi esnasında anahtarlama frekansı rezonans frekansına $(39680 \mathrm{~Hz})$ ayarlandığında evirici devre rezonans durumunda çalışmaktadır. Bu çalışma koşulları altında evirici akımının tepe değeri 5,55A'dir. Yapılan analiz ve benzetim çalışmasının sonuçlarında evirici akımının net görülebilmesi için $v_{a b}$ gerilimi $1 / 5$ oranında küçültülmüştür. Aynı devre parametreleri ile yapılan benzetim çalışmasının sonucu Şekil 4b'de sunulmuş ve evirici akımının tepe değeri 5,524A olarak ölçülmüş̧ür.

Analizi gerçekleştirilen diğer durum ise rezonans devre üzerindeki kondansatör geriliminin hesaplanmasıdır. RK-4 analizi sonucunda kondansatör gerilimi grafiği çizdirilmiş olup, Şekil 5a'da sunulmuştur. Buna göre kondansatör geriliminin tepe değeri 521,8V'dur. 


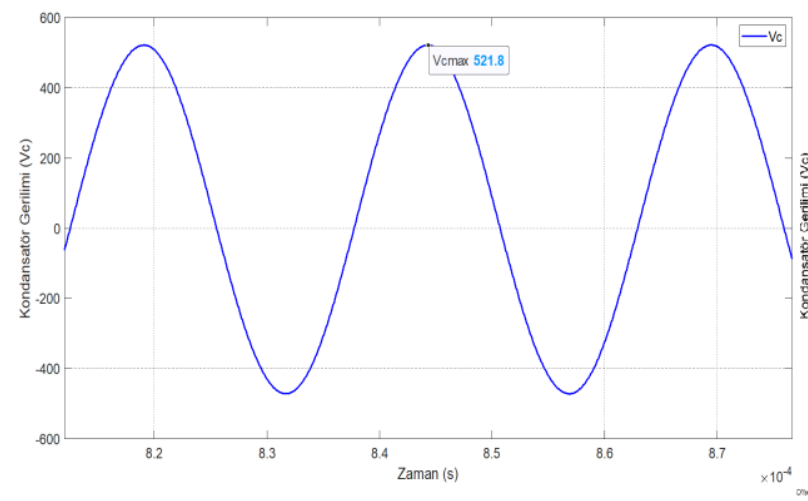

(a)

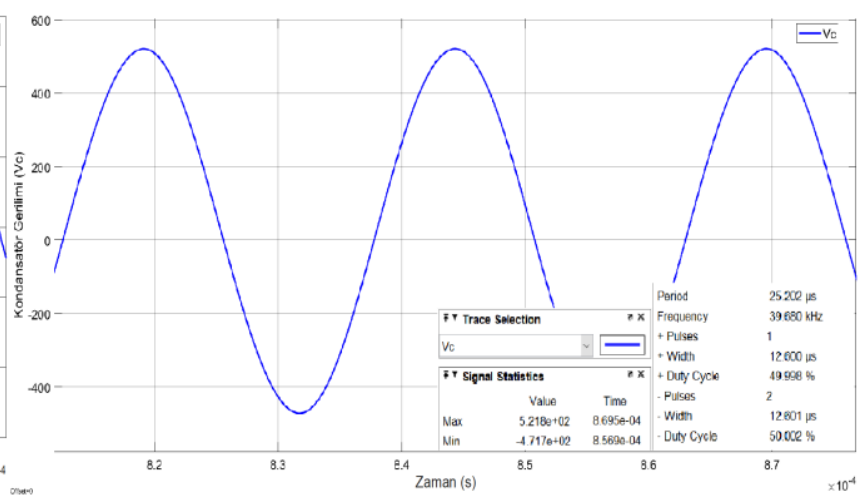

(b)

Şekil 5. Kondansatör Gerilimi (a) RK-4 Analizi Sonucu, (b) Benzetim Çalışması Sonucu

Benzetim çalışması sonucunda ise kondansatör geriliminin tepe değeri Şekil 5b'de görüldügü gibi 521,8V olarak ölçülmüştür.

\section{Sonuç}

D sınıfı seri rezonans eviriciler yüksek güçleri kontrol edebilmeleri ve tam köprü eviricilere göre daha az devre elemanı gerektirmeleri nedeniyle tercih edilmektedir. Bu eviricilerin analizinde ikinci dereceden diferansiyel denklemler ortaya çıkmaktadır. İkinci dereceden diferansiyel denklemin çözümü için Euler, Heun ve Runge-Kutta gibi çeşitli analiz yöntemleri mevcuttur. Bu çalışmada analiz yöntemlerinden daha güvenilir sonuçlar verdiği için Klasik Runge-Kutta yöntemi kullanılmıştır. Bu yöntem MATLAB ortamında yazılan bir algoritma ile gerçekleştirilmiştir. Aynı zamanda MATLAB/Simulink ortamında da aynı devre parametreleri ile benzetim çalışması gerçekleştirilerek yazılan algoritmanın doğruluğu test edilmiştir. Yazılan RK-4 algoritmasının hızlı, doğru ve güvenilir sonuçlar verdiği gözlemlenmiştir. İlerideki çalışmalarda güvenilir sonuç veren RK-4 algoritması farklı yapılardaki güç dönüştürücü devrelerinin analizi gerçekleştirilebilir. Aynı zamanda deneysel çalışmalardan elde edilecek sonuçlarla RK-4 algoritması ve diğer analiz yöntemleri karşılaştırılabilir.

\section{Kaynaklar}

[1] Y.-C. Chuang, Y. Ke, H. Chuang, Jung-Tai Chen, A novel loaded-resonant converter for the application of DC-to-DC energy conversions, IEEE Ind. Appl. Soc. Annu. Meet., IEEE, 2011; 1-8.

[2] B. Guo, S. Dwari, S. Priya, Voltage-Controlled Tunable Capacitor based Resonant Power Converter, IEEE Energy Convers. Congr. Expo., IEEE, 2019; 2164-2169. 
[3] H. Cha, F.Z. Peng, D. Yoo, Z-source resonant DC-DC converter for wide input voltage and load variation, Int. Power Electron. Conf. - ECCE ASIA -, IEEE, 2010; 995-1000.

[4] S. Nacar, S. Öncü, Hydrogen production system with fuzzy logic-controlled converter, TURKISH J. Electr. Eng. Comput. Sci. 2019; 1885-1895.

[5] M. Arazi, A. Payman, M.B. Camara, B. Dakyo, Study of different topologies of DC-DC resonant converters for renewable energy applications, Thirteen. Int. Conf. Ecol. Veh. Renew. Energies, IEEE, 2018; 1-6.

[6] I. Song, D. Jung, Y. Ji, S. Choi, Y. Jung, C. Won, A soft switching boost converter using an auxiliary resonant circuit for a PV system, 8th Int. Conf. Power Electron. - ECCE Asia, IEEE, 2011; 2838-2843.

[7] Dong-Woo Han, Hee-Jun Lee, Soo-Cheol Shin, Jun-Gu Kim, Y.-C. Jung, C.-Y. Won, A new soft switching ZVT boost converter using auxiliary resonant circuit, IEEE Veh. Power Propuls. Conf., IEEE, 2012; 1250-1255.

[8] M. Salem, A. Jusoh, N.R.N. Idris, I. Alhamrouni, Performance study of series resonant converter using zero voltage switching, IEEE Conf. Energy Convers., IEEE, 2014; 96100 .

[9] N. Öztürk, Sert ve Yumuşak Anahtarlamalı Tam Köğrü Çeviricilerde Kayıp Analizi, Gazi Üniv. Müh. Mim. Fak. Der. 2008 147-155.

[10] O. Henry, B. Albert, I. Justice, Application of Numerical Methods in Transient Analysis, Int. J. Sci. Res. 2019; 2108-2112.

[11] T.A. Kee, R. Ranom, Comparision of Numerical Techniques in Solving Transient Analysis of Electrical Circuits, ARPN J. Eng. Appl. Sci. 2018; 314-320.

[12] I.S.M. Zawawi, H. Aris, B.N. Jørgensen, Transient Analysis of Electrical Circuits using Block Backward Differentiation Formula, 9th Int. Conf. Informatics, Environ. Energy Appl., 2020; 99-103.

[13] M.K. Kazimierczuk, D. Czarkowski, Resonant Power Converters, Second Edi, John Wiley \& Sons Inc., Canada, 2011.

[14] H. Özbay, PDM-MPPT based solar powered induction heating system, Eng. Sci. Technol. 
an Int. J. 2020.

[15] S.C. Chapra, Applied Numerical Methods with MATLAB, 3rd., McGraw-Hill, Natick, MA, 2012. 